\title{
A Survey on Anomaly Detection and Diagnosis Problem in the Space System Operation
}

\author{
Seçil Taburoğlu* \\ Senior Software Design Engineer Turkish Aerospace Industries Ankara, Türkiye
}

\begin{abstract}
Spacecraft telemetry data is transferred from satellite to ground control station. The data contains not only information about health status of the satellite but also contains response messages to telecommand (telecommand data is send to spacecraft from ground control station) data. Telemetry data can indicate data error, communication link failure, sensor error, equipment and electronic devices failure. Safety and reliability are provided by telemetry and telecommand data. The most important subjects are safety and reliability for space mission. Therefore, telemetry data should be analyzed and take measures against to attack or unexpected situation. Various intelligent anomaly detection methods are proposed in the literature. Supervised/unsupervised (machine learning) anomaly detection approaches and data mining technology are the most used methods. This paper is a literature review for anomaly detection approaches in space system operation. Anomaly detection techniques have been investigated in the literature, but studies on space domain is quite limited. It is considered to contribute to literature in terms of that.
\end{abstract}

Keywords: Anomaly detection; Machine learning, Data mining; Spacecraft health.

\section{Uydu Sistemlerinde Anomali Tespiti ve Tanısı Sorununa Yönelik Yapılmış Bir}

\section{Araștırma}

Öz

Uydu uzölçüm verileri uydudan yer kontrol istayonuna transfer edilir. Bu veri sadece uydu sağlık verilerini içermekle kalmayıp uzkomut (telekomut verisi, yer kontrol istasyonundan uyduya iletilir) mesajlarına yanıtları da içerir. Telemetri verileri; veri hatası, iletişim bağlantısı hatası, sensör hatası, ekipman ve elektronik cihaz arızası gibi bilgileri içerebilir.Güvenlik ve güvenilirlik, telemetri ve telekomut verilerilerine bakılarak sağlanılmaktadır.Uzay görevinde en önemli konular güvenlik ve güvenilirlik olduğu için telemetri verileri analiz edilmeli ve saldırılara veya beklenmeyen durumlara karşı önlemler alınmalıdır.Literatürde çeşitli akıllı anomali saptama yöntemleri önerilmiştir. Gözetimli/Gözetimsiz (makine öğrenmesi) anomali tespiti ve veri madenciliği yaklaşımları en çok kullanılan yöntemlerdir. Bu makale uzay sistemlerinde anomali tespiti hakkında genel bakış sunmaktadır.

Anahtar Kelimeler: Anomali tespiti; Makine öğrenmesi; Veri madenciliği;Uydu sağlı̆̆ı. 


\section{Introduction}

\section{A. Anomaly detection}

Anomaly detection (Chandola et al., 2009) is a technique used to identify dataset which does not conform to an expected behavior or other items in a dataset. In general, these unexpected behaviors are defined as attacks. Whereas these situations can be unexpected behaviors which are previously not known, rather than an attack.

The anomaly detection provides very significant and important information about the system. Also, anomaly detection helps prevent potential malfunctions and serious errors. This improves system security and reliability.

\section{B. Spacecraft anomaly detection}

Anomaly detection is an important topic for space system, because so much money and time are spent. Also satellites have become incredibly useful, especially for meteorology, communication, and navigation and military. These domains are costly and safety critical. Therefore failures are not acceptable. The anomaly detection can help carry out fault diagnosis and prevent the occurrence of potential failures.

There are many anomaly detection methods exist, in general following steps (Gilmore et al., 2016) are done.

- Data Preprocessing and Feature extraction: A list of related parameters for components or subsystems of the spacecraft system are selected

- Model Generating: Model is generated on the normal or abnormal behavior of the spacecraft system.

- Detecting: Statistical based, knowledge based or machine learning and data mining algorithms are used.

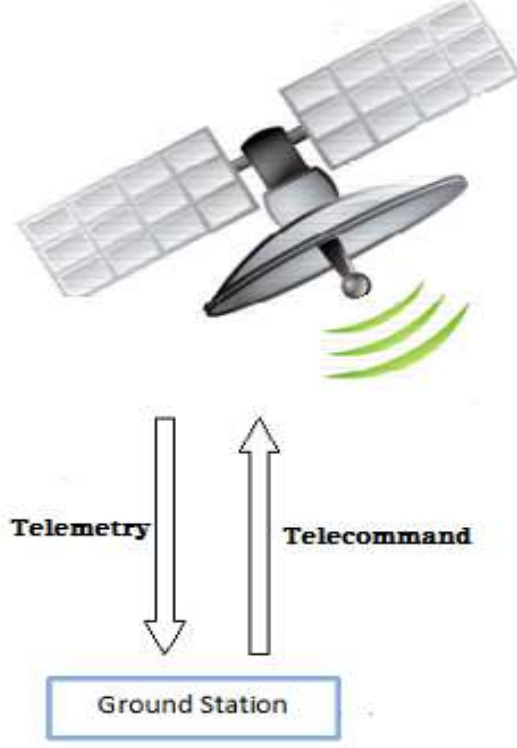

Figure 1 Telemetry - Telecommand Communications

Telemetry data include some sensor values, such as temperature, voltage, angular velocity and temperatures which have low and high limit value. That's why limit checking
(Machida et al., 2006) is one alternative for anomaly detection. But for some cases when limit values are normal, anomalies can exist, this means that some class of anomalies occur without violating the limits on the variables. In order to take measures before the situation occurs, these anomalies should be predicted. Also domain experts or operators always should monitor and review out of limit values. If the limit values are inappropriate, anomaly will detected, else false alarms will be generated and real anomalies may be missed. Therefore, to overcome these problems machine learning methods are used instead of limit checking. Also, Machida, K., (Machida et al., 2006) have developed a new method for limit checking. They have combined limit checking and Sparse Bayesian Learning (or Relevance Vector Machine). RVM is used to learn a model of high and low limit values from old normal telemetry. The resulting models are then used in later operations to detect anomalies for target variables online.

Data Mining Techniques (clustering and classification based) and hybrid approach are the most important methods. An important advantage of these approaches compared with the expert systems and model-based approach is that it does not require complete and accurate expert knowledge or models.

There are a large number of surveys in the literature about the detection of anomaly but there is very limited number of in terms of space domain. This survey aims to give a closer look on these enhancements and to summarize and categorize some articles presented in this field according to the various anomaly detection techniques. Also, in this review, the methods in the literature are shortly summarized for the industry (real world) which have just started to work on the anomalies in the spacecraft.

\section{Methodology}

There are two main approach for anomaly detection: knowledge-driven approach and data-driven approach (Yairi et al., 2017). In knowledge-driven approach, highly accurate results can be obtained, if knowledge is accurate. However, this method is costly because it require expensive expert knowledge and manual checking. Data-driven approach includes machine learning methods (Liu, D et al., 2017; Biswas et al., 2016; Gao et al., 2012a; Machida et al., 2006; Shi, et al., 2017; Azevedo et al., 2012) (classification (Liu et al., 2017; Machida et al., 2006; Gao et al., 2012), clustering (Biswas et al., 2016; Gao et al., 2012; Shi et al., 2017; Azevedo et al., 2012)). This approach is better than knowledge-driven in terms of cost. Also it is an automatic detection approach; however, most of the time highly accurate results are not available.

\section{Machine Learning Approaches (Data-driven approaches)}

Reviewed methods are listed in Table 1. This table includes dataset and methods' pros and cons.

\section{A. Classification approaches (supervised learning)}

Labelled data is required for classification. Some of the data is used for training and labelled data is acquired on this method. 
This method classifies data divided into two categories as normal and abnormal. This approach is not fully automated and expert knowledge is required. In general, following algorithms are used to anomaly detection:

k-Nearest Neighbor (KNN) classification: KNN algorithm classifies data sets based on their similarity with neighbors. D. Liu, J. Pang (Liu, et al., 2017) have used KNN classification with enhanced similarity measures on actual telemetry data. Telemetry data has been divided into time series. Mahalanobis Distance and DTW Distance(Liu, et al., 2017) algorithm are used on telemetry data to calculate distance measure. Distance measure is used to measure the similarity of the telemetry data. KNN algorithm classifies new telemetry data according to the similarity measures. Liu, J. Pang (Liu et al., 2017), have obtained different results for different dataset. In general, they can obtain satisfied results with Mahalanobis Distance and DTW Distance.

Support Vector Machines (SVM): SVM supports classification and regression, which is useful for statistical learning theory, and fully recognizes these factors. SVM can be defined as a field of pattern recognition. SVM classifies data into two classes. Yu Gao, Tianshe Yang, Nan Xing (Gao et al., 2012) have used binary SVM to detect anomalies. This method is not valid for fault diagnosis because fault diagnosis has to deal with multi-type malfunctions. So, they have proposed two approach; combining several binary SVMs and implementing multi-class classification. They have verified approach with actual satellite data and simulated by Matlab/Simulink. They have obtained good results for two cases (accuracy: 99.2\%, 97.4\%), and performance is also satisfactory. Also they used Principal Component Analysis (PCA) for feature extraction. PCA is a dimension-reduction mathematical technique. This technique is used to reduce large datasets to smaller sets. Feature identification and reduction is an important step for anomaly detection.

\section{B. Clustering approaches (unsupervised learning)}

This method uses unlabeled telemetry data. Therefore there is no need for extra space domain knowledge. The object of unsupervised learning technique is to find similar objective data points and combines similar data points. Gao, Yu (Gao et al., 2012) have detected analogies in runtime by using unsupervised learning. Firstly, they automatically detects and removes abnormal data from the archived (historical) telemetry data to construct normal behavior model. Then, according to the normal behavior model. They have detected anomalies in real time data. Single-linkage clustering is used for detection. Single linkage is a type of hierarchical clustering methods. In this method, the similarity of the two clusters is the similarity of the most similar members. This study is important, because this proposed new approach does not require expert knowledge (low cost). Also, there is no cost of data training (labelled data). According to the results, when detection ratio increases, false positive ratio increases. Supervised learning results are higher than unsupervised learning results.
Azevedo, Denise Rotondi, Ana Maria Ambrósio, and Marco Vieira (Azevedo et al., 2012) have used K-means and Expectation Maximization(EM) for clustering. K-means is an unsupervised learning algorithm. This algorithm divides the data set with the number of groups represented by the variable K. EMalgorithm can be thought of as an extension of the k-means algorithm. EM is an iterative algorithm for learning probabilistic categorization model from unlabeled telemetry data. They have calculated the dissimilarity indexes using both Euclidean and Manhattan distances, then they have used clustering algorithms.

Fuertes, Sylvain, Barbara Pilastre, and Stéphane D'Escrivan (Fuertes et al., 2018) have compared three unsupervised algorithms: One-Class Support Vector Machine (OC-SVM is a type of Support Vector Machines) (Rana, Divya, 2015), Density-based spatial clustering of applications with noise (DBSCAN) and k-Nearest Neighbors algorithm. They have obtained approximate results. They argue that the success of the method depends on the choice of features rather than machine learning methods.

\section{Hybrid approaches}

Hybrid approach combines unsupervised and supervised methods. This approach is not fully automated, expert knowledge is required because of supervised learning techniques. The main goal is to develop more automated methods.

Biswas, Gautam (Biswas et al., 2016) have used unsupervised learning algorithm to cluster time series data. Their hypotheses is that the larger groups of clusters will show normal (routine telemetry data - maneuver, expected orbit propagation etc.) operations. On the other hand smaller groups will show anomalous situations. They have used hierarchical clustering method. Then they have verified results by a supervised approach of consulting domain experts. Although they achieve good results, this method is not automate and cheap.

Machida, K. (Machida et al., 2006) has used Dynamic Bayesian Network(DBN) to learn model from past telemetry data in normal operation by Expectation-Maximization algorithm. It is particularly suited for modeling hybrid systems involving both continuous variables and discrete variables. Also the DBN is well-suited for time-series space data.

\section{Other approaches}

Auto-Regressive Integrated Moving-Average (ARIMA) is used by Ibrahim, Sara K (Ibrahim et al., 2018). ARIMA is a model for time series forecasting. They have applied different machine learning techniques and compared to each other. According to the results, ARIMA have highest prediction accuracy but in case of short-term forecasting models. Also they have used PCA for dimension reduction but they lost some important data, consequently they preferred domain expert knowledge. 
Table 1 Articles classification

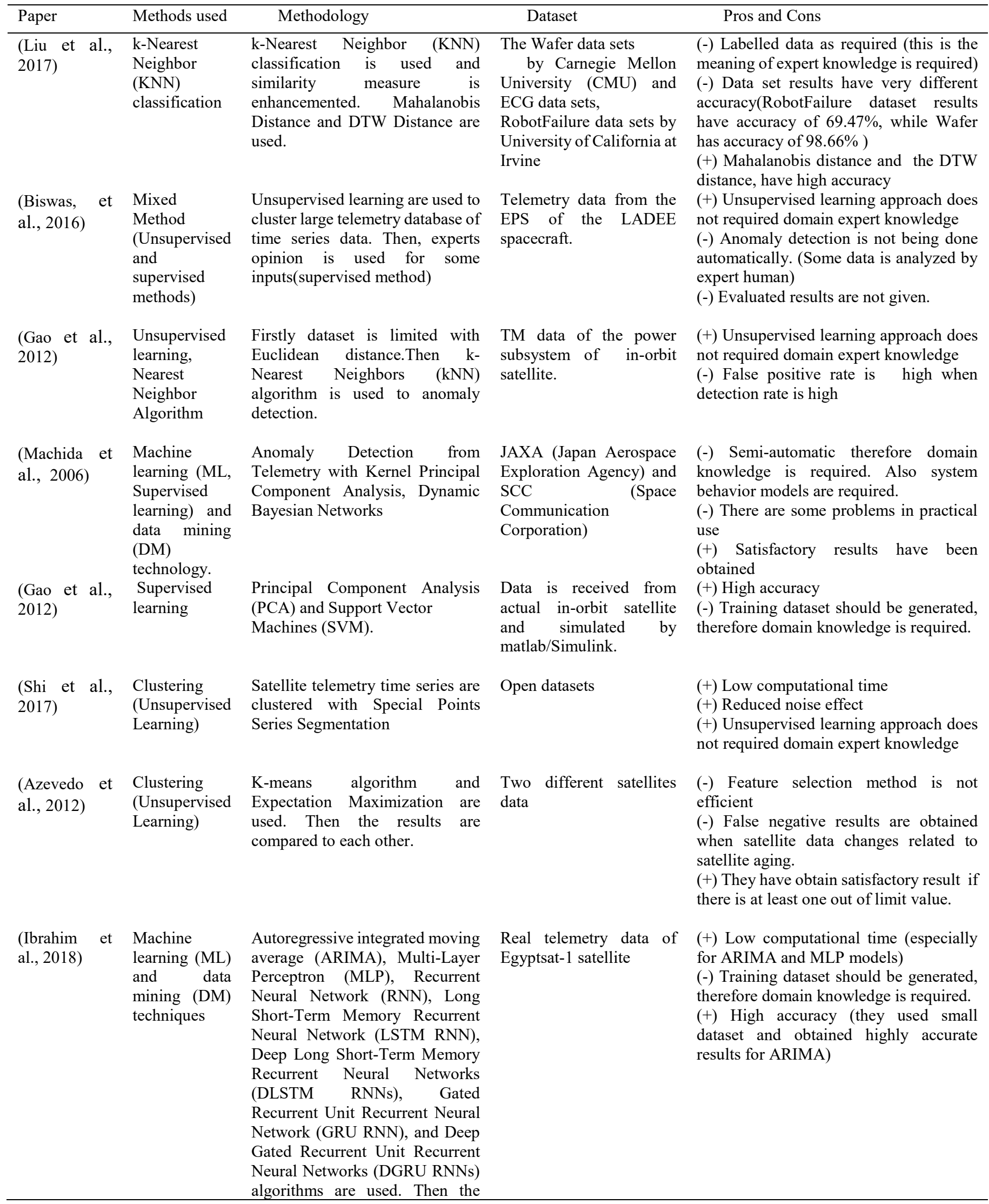


results are compared to each

other.

(Fuertes et Clustering One-Class Support Vector

al., 2018) (Unsupervised Machine (OC-SVM), k-Nearest Learning) Neighbors algorithm, DBSCAN
CNES operated satellite data
(+) High accuracy, but depends on the application context and feature selections.

\section{Conclusions}

The most critical and outstanding articles are reviewed for this survey. This survey shows the most used methods for anomaly detection in space domain. Through this literature review, several conclusions have been reached about anomaly detection and supervised/unsupervised learning algorithms for use in satellite anomaly detection system. Most of the proposed solutions are centered around data mining techniques. The work in fault analysis has mainly focused on supervised learning techniques. Naïve Bayes, Support Vector Machines and kNearest Neighbor (KNN) classification are the most frequently used machine learning algorithms. Nowadays supervised learning techniques are no longer used because they need knowledge of expertise. There is limited unsupervised study but the number of papers is increasing fast. Hybrid approaches are often used because they provide better results. However, there is a fact that abnormal detection systems still have high false positive rates and studies on this area still continues.

\section{References}

Azevedo, D.R., Ambrósio, A.M. and Vieira, M., 2012, May. Applying data mining for detecting anomalies in satellites. In 2012 Ninth European Dependable Computing Conference (pp. 212-217). IEEE..

Biswas, G., Khorasgani, H., Stanje, G., Dubey, A., Deb, S. and Ghoshal, S., 2016. An application of data driven anomaly identification to spacecraft telemetry data. In Prognostics and Health Management Conference.

Chandola, V., Banerjee, A. and Kumar, V., 2009. Anomaly detection: A survey. ACM computing surveys (CSUR), 41(3), p.15..

Fuertes, S., Pilastre, B. and D'Escrivan, S., 2018. Performance assessment of NOSTRADAMUS \& other machine learning-based telemetry monitoring systems on a spacecraft anomalies database. In 2018 SpaceOps Conference (p. 2559).

Gao, Y., Yang, T., Xing, N. and Xu, M., 2012, July. Fault detection and diagnosis for spacecraft using principal component analysis and support vector machines. In Industrial Electronics and Applications (ICIEA), 2012 7th IEEE Conference on (pp. 1984-1988). IEEE.

Gao, Y., Yang, T., Xu, M. and Xing, N., 2012, January. An unsupervised anomaly detection approach for spacecraft based on normal behavior clustering. In Intelligent Computation Technology and Automation (ICICTA), 2012 Fifth International Conference on (pp. 478-481). IEEE.

Gilmore, C. and Haydaman, J., 2016, January. Anomaly Detection and Machine Learning Methods for Network Intrusion Detection: an Industrially Focused Literature Review. In Proceedings of the International Conference on Security and Management (SAM) (p. 292). The Steering Committee of The World Congress in Computer Science,
Computer Engineering and Applied Computing (WorldComp).

Ibrahim, S.K., Ahmed, A., Zeidan, M.A.E. and Ziedan, I., 2018. Machine Learning Methods for Spacecraft Telemetry Mining. IEEE Transactions on Aerospace and Electronic Systems.

Liu, D., Pang, J., Xu, B., Liu, Z., Zhou, J. and Zhang, G., 2017, August. Satellite Telemetry Data Anomaly Detection with Hybrid Similarity Measures. In Sensing, Diagnostics, Prognostics, and Control (SDPC), 2017 International Conference on (pp. 591-596). IEEE.

Machida, K., Fujimak, R., Yairi, T., Kawahara, Y. and Sato, Y., 2006. Telemetry-mining: A machine Learning Approach to Anomaly detection and fault Diagnosis for space Systems. In 2nd IEEE International Conference on Space Mission Challenges for Information Technology, IEEE.

Rana, Divya, 2015, One Class SVM Vs SVM Classification Divya Rana.

Shi, X., Pang, J., Liu, D. and Peng, Y., 2017, July. Satellite telemetry time series clustering with improved key points series segmentation. In Prognostics and System Health Management Conference (PHM-Harbin), 2017 (pp. 1-7). IEEE.

Yairi, T., Takeishi, N., Oda, T., Nakajima, Y., Nishimura, N. and Takata, N., 2017. A Data-Driven Health Monitoring Method for Satellite Housekeeping Data Based on Probabilistic Clustering and Dimensionality Reduction. IEEE Transactions on Aerospace and Electronic Systems, 53(3), pp.1384-1401. 